\title{
Novel biomarkers may aid the decision for CT scan in emergency settings in mild head trauma
}

\author{
George A Alexiou ${ }^{1}$ (D), Georgios D Lianos ${ }^{*}{ }^{2}$, Athanasios Sotiropoulos ${ }^{1} \&$ Spyridon Voulgaris ${ }^{1}$ \\ ${ }^{1}$ Department of Neurosurgery, University Hospital of Ioannina, Ioannina, Greece \\ ${ }^{2}$ Department of Surgery, University Hospital of loannina, Ioannina, Greece \\ *Author for correspondence: Tel.: +30 694421 1005; georgiolianos@yahoo.gr \\ “Although computed tomography (CT) is indicative of moderate and severe head trauma, minor \\ head trauma constitutes a challenge, in differentiating patients in need for CT and those to be \\ reassured and discharged in the emergency department."
}

First draft submitted: 27 June 2019; Accepted for publication: 24 July 2019; Published online: 30 August 2019

Keywords: emergency setting • head injury • trauma

Traumatic brain injury (TBI) is an increasing public health problem and is one of the leading causes of death and disability worldwide annually. Although historically TBI is more common in young people, nowadays the mean age of patients with TBI is increasing. Older patients with TBI have several comorbidities and the use of anticoagulants and antiplatelet drugs is frequently in this age group. is caused primarily by falls and road injuries [1]. The majority of TBIs are mild in severity and constitute about $70-90 \%$ of all TBIs. In children, head injury is a frequent presentation to the emergency department. An incidence of 3419 cases per 100,000 population per year has been reported and $99 \%$ of these children had minimal head injury [2]. Children have several differences from adults in term of thinner skull, head is larger in proportion to the body, differences in the immune system and vulnerability to hypothermia. Furthermore, physicians should be aware that in children under 2 years of age child abuse remains a major cause of head trauma [3].

Although computed tomography (CT) is indicative of moderate and severe head trauma, minor head trauma constitutes a challenge, in differentiating patients in need for $\mathrm{CT}$ and those to be reassured and discharged in the emergency department. The primary motivation for performing a CT is the exclusion of an intracranial hemorrhage with potentially catastrophic sequelae. The use of CT for mild TBI has increased over the last years, despite of its low diagnostic yield. In children this practice is especially associated with an increased lifetime risk of malignancy due to ionizing radiation [4]. In a study of 10.9 million children and adolescents that underwent a diagnostic CT scan, 60,674 cancer cases were diagnosed after a mean follow-up of 9.5 years. The exposed people had $24 \%$ greater cancer incidence than unexposed people [5]. To date, several sets of clinical decision rules have been developed for children, and showed good accuracy in identifing clinically important head injuries. These include he Pediatric Emergency Care Applied Research Network (PECARN); Canadian Assessment of Tomography for Childhood Head injury (CATCH) and the Children's Head injury ALgorithm for the prediction of Important Clinical Events (CHALICE) [6] - however, in adults the impact of decision rules on CT use is limited [7]. On the other hand, rapid MRI protocols of 3-4 min duration have been recommended for acute head injury; however, utility of MRI acutely is limited [8]. MRI mainly misses skull fractures whereas CT scan mainly miss diffuse axonal injury, subarachnoid hemorrhage and small subdural hematomas [8]. Overall MRI might be useful for moderate or severe TBI in which follow-up scans are required, but for minor TBI a cost-effectiveness analysis should be performed. Finally, the unnecessary CT-associated cost is high. Thus, TBI-specific and reliable biomarkers are urgently needed. A useful biomarker should have high sensitivity and specificity for the identification of clinical important TBI, should be readily measured (i.e., in blood, cerebrospinal fluid) and of low cost in order to be widely available. 
TBI has been associated with several pathophysiological events and processes, and is responsible for primary and secondary brain damage. To date many biomarkers have been investigated; however, limited guidelines exist regarding their use. These biomarkers are usually derived from different elements of the injured brain, such as dendritic injury, axonal injury and neuronal cell body injury [9]. UCH-L1 is expressed in neurons and GFAP is an astroglial biomarker. Both have been found elevated after head trauma. Recently in a prospective, multicenter observational study both UCH-L1 and GFAP were measured in serum and analyzed using prespecified cutoff values in mild and moderate TBI patients within $12 \mathrm{~h}$ of injury. The test exhibited a sensitivity of $97.6 \%$ and a negative predictive value of $99.6 \%$, whereas in only $3 / 1959$ patients, the test was negative when the CT scan showed positive results [10]. The US FDA recently approved the first blood test to evaluate mild TBI based on UCH-L1 and GFAP levels. S-100B is another astroglial biomarker and is the most well studied as an indicator of intracranial injury and postconcussive syndrome. In a study of 726 patients with mild head trauma, in which 29 patients had positive CT findings, the measurement of S-100B proved cost effective and reduced CT usage [11]. However, S100B increases in other types of trauma without TBI and has several shortcomings in predicting outcome after mild TBI. MBP tau promotes microtubule assembly and stability of neurons. Accumulation of the abnormal hyperphosphorylated tau (P-tau) protein can be found after TBI and in neurodegenerative diseases such as Alzheimer's disease. A study of 217 patients with TBI assessed the plasma P-tau levels, total tau protein (T-tau) levels and P-tau/T-tau ratio as a diagnostic and prognostic tool. Compared with T-tau levels alone, P-tau levels and P-tau/T-tau ratios were better markers in distinguishing patients with positive CT findings. A correlation was also found between P-tau levels and P-tau/T-tau ratio with patient outcomes [12]. Other biomarkers that have been studied and showed some promise were NSE, MMP-2, CK-BB, neurofilament proteins and MBP; however, further studies are needed [9].

Increased blood glucose levels have been reported after head trauma and glucose levels over $200 \mathrm{mg} / \mathrm{dl}$ have been associated with increased mortality rate. Hyperglycemia is more common in severe TBI. Several mechanisms by which hyperglycemia is induced after TBI have been reported so far [13]. Stress induced by TBI increases stress hormones levels, such as catecholamine, glucagon, cortisol and growth hormone, which in turn increase blood glucose levels. Systemic inflammatory response syndrome is also frequently encountered after TBI and can be a cause of hyperglycemia via cytokines and CRH induction. Post traumatic hypopituitarism may influence glucose homeostasis and finally some iatrogenic factors may cause hyperglycemia [13]. In a study that included 159 patients with mild TBI, the patients with positive CT findings had significant higher glucose levels than patients with negative CT findings. A serum glucose cut-off value of $120 \mathrm{mg} / \mathrm{dl}$ discriminated patients with positive CT findings with a sensitivity of $74.4 \%$ and a specificity of $90.7 \%$. It is of interest that all patients with blood glucose levels over $128 \mathrm{mg} / \mathrm{dl}$ had positive CT findings. A score of 14 in the Glasgow Coma Scale (GCS) had 27.2\% sensitivity and 95.6\% specificity for the detection of patients with positive CT findings. Furthermore, positive CT findings were more common in patients presented with history of loss of consciousness and post traumatic amnesia. However, no significant difference was found in glucose levels between patients with and without alcohol intoxication [14]. Increased blood glucose levels have been also correlated with coagulopathy occurrence. A serum glucose cut-off value of $151 \mathrm{mg} / \mathrm{dl}$ or higher could detect patients that developed coagulopathy with a sensitivity of $91.5 \%$ and a specificity of $87.5 \%$. Furthermore, patients with hemorrhagic contusions and midline shift at admission had significant higher blood glucose levels. Apart from glucose levels, hemoglobin levels lower than $12.4 \mathrm{mg} / \mathrm{dl}$ were significantly associated with coagulopathy occurrence [15]. Coagulopathy after head trauma is a common finding and its occurrence is associated with TBI severity, being more common in severe TBI. Coagulopathy occurrence has a negative impact on secondary brain damage and is also associated with increased morbidity and mortality. Since, blood glucose levels assessment is inexpensive and routinely performed, it would be interesting that this test to be combined with other TBI biomarkers in order to increase sensitivity and specificity. Furthermore, studies are needed to address whether timely treatment of hyperglycemia has any effect on outcome of TBI patients.

In conclusion, the identification and clinical use of novel biomarkers for predicting the need for CT in mild TBI are urgently needed. This is important, apart from avoiding the radiation exposure to the patients, for reducing excessive cost, waiting hours in the emergency department, when CT is not readily available, and the false positive findings that have to be checked out and over diagnosis. Furthermore, reliable biomarkers may allow for classifying TBI patients, predicting outcome and coagulopathy occurrence, permitting investigational therapies to be evaluated or monitor drug efficiency. Although hypothetically a combination of biomarkers may increase accuracy for the identification of clinical important TBI, this needs to be proven and a cost-effectiveness analysis should also be performed. Furthermore, one should investigate how these biomarkers work in clinical setting, if their accuracy differs according to GCS score and to quantify the reduction of head CT use that they may produce. Finally, setting 
cut-off values needs to be more carefully evaluated and investigate differences between patients just or high above the cut-off values. Certainly more studies are needed, as well as studies in pediatric population, in which mild head trauma is very frequent.

\section{Financial \& competing interests disclosure}

The authors have no relevant affiliations or financial involvement with any organization or entity with a financial interest in or financial conflict with the subject matter or materials discussed in the manuscript. This includes employment, consultancies, honoraria, stock ownership or options, expert testimony, grants or patents received or pending, or royalties.

No writing assistance was utilized in the production of this manuscript.

\section{References}

1. GBD 2016 Neurology Collaborators. Global, regional, and national burden of neurological disorders, 1990-2016: a systematic analysis for the Global Burden of Disease Study 2016. Lancet. Neurol. 18(5), 459-480 (2019).

2. Hawley C, Wilson J, Hickson C et al. Epidemiology of paediatric minor head injury: comparison of injury characteristics with Indices of Multiple Deprivation. Injury 44(12), 1855-1861 (2013).

3. Alexiou GA, Sfakianos G, Prodromou N. Pediatric head trauma. J. Emerg. Trauma Shock 4(3), 403-408 (2011).

4. Pearce MS, Salotti JA, Little MP et al. Radiation exposure from CT scans in childhood and subsequent risk of leukaemia and brain tumours: a retrospective cohort study. Lancet 380(9840), 499-505 (2012).

5. Mathews JD, Forsythe AV, Brady Z et al. Cancer risk in 680,000 people exposed to computed tomography scans in childhood or adolescence: data linkage study of 11 million Australians. BMJ 346, f2360 (2013).

6. Babl FE, Borland ML, Phillips N et al. Pediatric Research in Emergency Departments International Collaborative (PREDICT). Accuracy of PECARN, CATCH, and CHALICE head injury decision rules in children: a prospective cohort study. Lancet 389(10087), 2393-2402 (2017).

7. Sharp AL, Nagaraj G, Rippberger EJ et al. Computed tomography use for adults with head injury: describing likely avoidable emergency department imaging based on the Canadian CT head rule. Acad. Emerg. Med. 24(1), 22-30 (2017).

8. Roguski M, Morel B, Sweeney M et al. Magnetic resonance imaging as an alternative to computed tomography in select patients with traumatic brain injury: a retrospective comparison. J. Neurosurg. Pediatr. 15(5), 529-534 (2015).

9. Wang KK, Yang Z, Zhu T et al. An update on diagnostic and prognostic biomarkers for traumatic brain injury. Expert. Rev. Mol. Diagn. 18(2), 165-180 (2018).

10. Bazarian JJ, Biberthaler P, Welch RD et al. Serum GFAP and UCH-L1 for prediction of absence of intracranial injuries on head CT (ALERT-TBI): a multicentre observational study. Lancet. Neurol. 17(9), 782-789 (2018).

11. Calcagnile O, Anell A, Undén J. The addition of S100B to guidelines for management of mild head injury is potentially cost saving. BMC Neurol. 16(1), 200 (2016).

12. Rubenstein R, Chang B, Yue JK et al. Comparing plasma phospho tau, total tau, and phospho tau-total tau ratio as acute and chronic traumatic brain injury biomarkers. JAMA Neurol. 74(9), 1063-1072 (2017).

13. Shi J, Dong B, Mao Y et al. Review: traumatic brain injury and hyperglycemia, a potentially modifiable risk factor. Oncotarget 7(43), 71052-71061 (2016).

14. Alexiou GA, Sotiropoulos A, Lianos GD et al. Blood glucose levels may aid the decision for CT scan in minor head trauma. Dis. Markers 2019, 1065254 (2019).

15. Alexiou GA, Lianos G, Fotakopoulos G, Michos E, Pachatouridis D, Voulgaris S. Admission glucose and coagulopathy occurrence in patients with traumatic brain injury. Brain Inj. 28(4), 438-441 (2014). 
